\title{
Mitochondrial genome in Hypsizygus marmoreus and its evolution in Dikarya
}

\author{
Gang Wang ${ }^{1,2 \dagger}$, Jingxian $\mathrm{Lin}^{3 \dagger}$, Yang Shi ${ }^{3}$, Xiaoguang Chang ${ }^{2}$, Yuanyuan Wang ${ }^{2}$, Lin Guo ${ }^{2}$, Wenhui Wang ${ }^{3}$, \\ Meijie Dou ${ }^{3}$, Youjin Deng ${ }^{1,2}$, Ray Ming ${ }^{1,4}$ and Jisen Zhang ${ }^{1 *}$ (i)
}

\begin{abstract}
Background: Hypsizygus marmoreus, a high value commercialized edible mushroom is widely cultivated in East Asia, and has become one of the most popular edible mushrooms because of its rich nutritional and medicinal value. Mitochondria are vital organelles, and play various essential roles in eukaryotic cells.

Results: In this study, we provide the Hypsizygus marmoreus mitochondrial (mt) genome assembly: the circular sequence is 102,752 bp in size and contains 15 putative protein-coding genes, 2 ribosomal RNAs subunits and 28 tRNAs. We compared the mt genomes of the 27 fungal species in the Pezizomycotina and Basidiomycotina subphyla, with the results revealing that $\mathrm{H}$. marmoreus is a sister to Tricholoma matsutake and the phylogenetic distribution of this fungus based on the mt genome. Phylogenetic analysis shows that Ascomycetes mitochondria started to diverge earlier than that of Basidiomycetes and supported the robustness of the hyper metric tree. The fungal sequences are highly polymorphic and gene order varies significantly in the dikarya data set, suggesting a correlation between the gene order and divergence time in the fungi mt genome. To detect the mt genome variations in $\mathrm{H}$. marmoreus, we analyzed the mtDNA sequences of 48 strains. The phylogeny and variation sited type statistics of $\mathrm{H}$. marmoreus provide clear-cut evidence for the existence of four well-defined cultivations isolated lineages, suggesting female ancestor origin of $\mathrm{H}$. marmoreus. Furthermore, variations on two loci were further identified to be molecular markers for distinguishing the subgroup containing 32 strains of other strains. Fifteen conserved protein-coding genes of mtDNAs were analyzed, with fourteen revealed to be under purifying selection in the examined fungal species, suggesting the rapid evolution was caused by positive selection of this gene.
\end{abstract}

Conclusions: Our studies have provided new reference mt genomes and comparisons between species and intraspecies with other strains, and provided future perspectives for assessing diversity and origin of $\mathrm{H}$. marmoreus.

Keywords: Hypsizygus marmoreus, Basidiomycota, Mitochondrial genome, SNP, Dikarya

\section{Introduction}

Mitochondria (mt) are vital organelles, and play various essential roles in eukaryotic cells. Since the discovery of mt DNA in 1963, large-scale studies have been performed to analyze their structures and functions. The evolution of the $\mathrm{mt}$ genome is fundamentally different from the major groups of eukaryotes (animals, plants, protists and fungi) [1]. Plant $\mathrm{mt}$ genomes have high recombination

\footnotetext{
* Correspondence: zjisen@126.com; zjisen@fafu.edu.cn

+ Gang Wang and Jingxian Lin contributed equally to this work.

${ }^{1}$ Center for Genomics and Biotechnology, Haixia Institute of Science and

Technology, Fujian Provincial Key Laboratory of Haixia Applied Plant Systems

Biology, College of Life Sciences, Fujian Agriculture and Forestry University,

Fuzhou 350002, China

Full list of author information is available at the end of the article
}

frequencies, including large intergenic regions, introns and their associated intronic open reading frames (ORFs) [2], and the repetitive genomic elements, which therefore causes variations in the mitochondrial $(\mathrm{mt})$ genome size [3] as seen for example, in the Silene genus. In contrast, animal mt genomes tend to have higher rates of DNA sequence evolution than in plants [4], and in general their $\mathrm{mt}$ genomes are gene rich and have fewer introns $[5,6]$. Historically compared to animals and plants, fungal mt genomes have been studied less. With the emergence of sequencing technology in recent years, a continuously increasing number of fungal $\mathrm{mt}$ genomes is analyzed, and about 186 complete fungal $\mathrm{mt}$ genomes are available at NCBI as of July 2019. This provides a powerful resource

(c) The Author(s). 2019 Open Access This article is distributed under the terms of the Creative Commons Attribution 4.0 International License (http://creativecommons.org/licenses/by/4.0/), which permits unrestricted use, distribution, and 
for comparative studies to reveal the patterns and mechanisms of mt genome evolution [7].

The fungal mt genome provides important clues to fungal evolution, population genetics and biology because it displays remarkable variations in terms of gene order, genome size, composition of intergenic regions, presence of repeats, introns, and associated ORFs [1, 8]. Previous studies revealed that the genome size of mitochondria ranged from $19 \mathrm{~kb}$ to $235 \mathrm{~kb}[9,10]$, which mainly result from the presence or absence of large intronic and intergenic sequences [11] with the number and length of introns being the most predominant factors [12]. One hypothesis suggested that introns were abundant in the ancestral $\mathrm{mt}$ genes, and were subsequently lost in most lineages [13]. The mt genomes of conspecific fungi species may also be divergent as indicated through the analysis of $\mathrm{mt}$ genomes of 11 Cordyceps militaris strains, the sizes of these $\mathrm{mt}$ genomes varied from 26.5 to $33.9 \mathrm{~kb}$ and the number of introns in genes ranged from two to eight [14]. Mt molecular markers have been successfully applied for evolutionary biology and systematics because $\mathrm{mt}$ genomes often evolve faster than nuclear genomes and allow for robust phylogenetic analyses based on the conserved proteins of the oxidative phosphorylation system [15].

In this study, a commercial strain of H. marmoreus was sequenced using PacBio sequencing, and a complete $\mathrm{mt}$ genome of $102,752 \mathrm{bp}$ was generated. The aims at this study are to (i) present a complete and annotated $\mathrm{mt}$ genome sequence of $H$. marmoreus, (ii) compare the mt genome of $H$. marmoreus with the genomes of other fungi in Pezizomycotina and Basidiomycotina to identify the common and specific characteristics of the mt genomes, (iii) provide insights into the evolution and phylogenetic relationships between different strains of $H$. marmoreus through the analysis of the variations at specific loci.

\section{Results}

\section{Sequence and general features of the $H$. marmoreus $\mathrm{mt}$} genome

Based on sequence homology with Pleurotus ostreatus $\mathrm{mt}$ genome (GenBank:NC_009905) we identified a scaffold with the length of $102,752 \mathrm{bp}$ as $\mathrm{mt}$ genome. The complete mt genome of $H$. marmoreus is a circular DNA molecule with a GC content of $32 \%$, containing 56 annotated genes (Table 1, Fig. 1). Of the 56 genes, fifteen genes are conserved protein-coding genes, with known functions in the electron transport chain and oxidative respiration. Seven NADH dehydrogenases (nad1, nad2, nad3, nad4, nad4L, nad5, nad6) are subunits of complex I [16]; cob is one subunit of coenzyme Q-cytochrome c reductase, which plays a critical role in the biochemical generation of ATP [17]; three cytochrome $c$ oxidases (complex IV: coxl, cox2, cox3) are the last enzymes in the respiratory electron transport chain [18]; three ATP synthase subunits (atp6, atp8, atp9) [11], and a ribosomal protein $\mathrm{S} 3$ gene, rps3 which is known to play critical roles in ribosome biogenesis and DNA repair. In addition, twenty-nine non-coding genes, including 27 tRNA and the small and large ribosomal RNA subunits (rns, rnl), were identified. Twelve free-standing ORFs were divided into two categories, eight ORFs in intronic sequences and 4 ORFs in intergenic regions.

The $48 \mathrm{H}$. marmoreus dikaryotic strains were sequenced with the Illumina Hiseq2500 platform, and the short reads were aligned to the reference genome to identify variants that were isolated by both GATK and SAMtools [19]. Through the selection of the overlaps from the two methods and a hard filtering step with stringent thresholds, 1373 reliable variant sites including 972 SNPs and 401 InDels, were identified in the mt genome sequence (Fig. 1, Additional file 9: Table S3). According to the SNPs pattern of 48 strains on the SNP loci (Fig. 2, Additional file 10: Table S4, Additional file 11: Table S5), 6964 genotype sites of $48 \mathrm{H}$. marmoreus strains were detected to be homozygous and different to the reference genome. While only 281 sites were detected to be heterozygous, a high ratio of up to $96.12 \%$ of the genotypes was homozygous, indicating that the $\mathrm{mt}$ genome of $H$. marmoreus existing in dikaryotic cells was a haplotype. A phylogenic tree was constructed based on the $972 \mathrm{mt}$ SNPs from 48 strains with the bootstrap value of 1000 times (Fig. 2, Additional file 3: Figure S3). The phylogenetic tree clearly showed the existence of four clusters that diverged from each other. According to the classification of the cultivated species on the tree, the $H$. marmoreus was divided into three groups (group I, II, III, IV). Group I contained 32 strains with the low bootstrap values, indicating that the mt genomes of these strains are highly conserved, whereas, strain HM54, is an exception, and was separated from all other strains.

Moreover, we performed PCR amplification of $\mathrm{mt}$ DNA based on two InDels (Additional file 13: Table S7): one (GGGGTCCCGTAC/G) located at position 93,344 on the mt genome and another (TAGTAA/T) located at position 93,608 of the $\mathrm{mt}$ genome, could be used as molecular markers to differentiate the group I from the other groups, verifying the reliability of the mt DNA for the classification of $H$. marmoreus stains.

\section{Annotation of variants and neutral evolution of the $\mathrm{mt}$ genome}

Through analysis using snpEFF software, 1942 effects were detected to be caused by 1373 variants (Table 2). Most of the variants $(83 \%)$ were located in the intergenic region (including the upstream and downstream regions of the gene); of the $17 \%$ remaining, 10 and $7 \%$ are located in the exon region and intron of the gene, respectively. Among the 


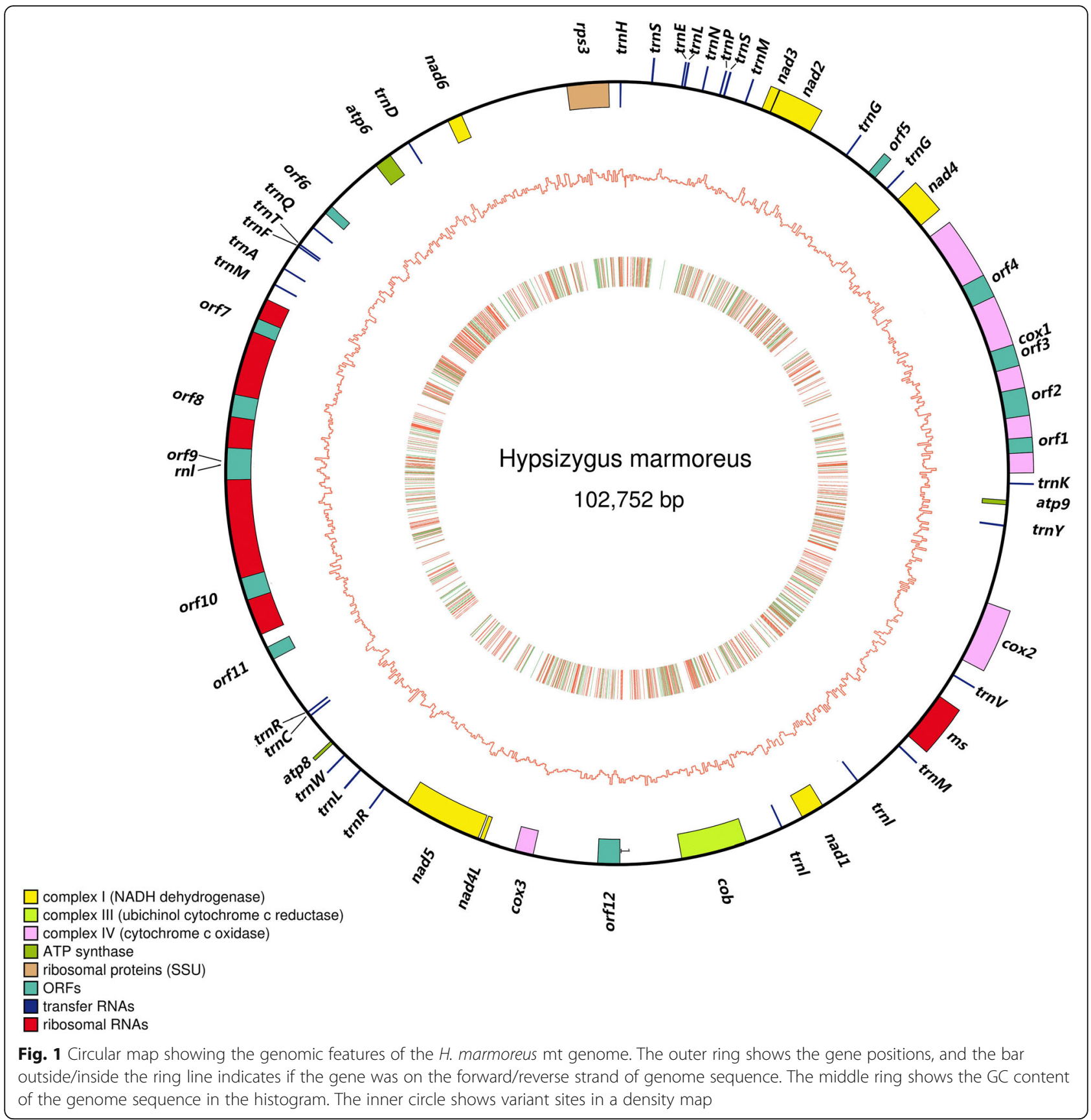

variants in codons of protein-coding genes, 18 nonsynonymous $(\mathrm{dN})$ mutation sites and 50 synonymous $(\mathrm{dS})$ mutation sites were observed. The ratio of nonsynonymous to synonymous mutations (dN/dS) was 0.36 , much less than 1 , indicating that, the general, $\mathrm{mt}$ homologous genes were in general undergoing purification selection during the evolutionary process. Of note, genes atp6, cob, cox1, nad2, nad4L and nad5 have a synonymous mutations number $>=3$ (Additional file 12: Table S6). Interestingly, the $\mathrm{dN} / \mathrm{dS}$ ratio of the rps3 gene has a value of 1.4 (7/5), suggesting rapid evolution was caused by the positive selection of this gene, which may explain the phenomenon that the rps3 gene was not found in part of other fungal species, such as, P. ostreatus, T. cingulata [1]. Frameshift mutations can cause overall changes in the protein sequences and are a type of mutation that has the greatest impact on gene function [20]. Five high impact variant sites were predicted by the SnpEFF software, including three frameshift variants in rps3, one frameshift variant in nad $4 L$ and the loss of 1 stop codon in nad5 (Table 3, 
Table 1 Summary of 56 genes of the $H$. marmoreus mt genome

\begin{tabular}{|c|c|c|c|}
\hline $\begin{array}{l}\text { Gene } \\
\text { category }\end{array}$ & Gene family & $\begin{array}{l}\text { Genes } \\
\text { number }\end{array}$ & Gene names \\
\hline \multirow[t]{5}{*}{ Conserved gene } & $\begin{array}{l}\mathrm{NADH} \\
\text { dehydrogenase subunit }\end{array}$ & 7 & nad1,nad2,nad3,nad4,nad4L,nad5, nad6 \\
\hline & $\begin{array}{l}\text { Cytochrome c oxidase } \\
\text { subunit }\end{array}$ & 3 & $\operatorname{cox} 1, \operatorname{cox} 2, \operatorname{cox} 3$ \\
\hline & ATP synthase subunit & 3 & atp6, atp8, atp9 \\
\hline & Apo cytochrome b & 1 & Cob \\
\hline & Ribosomal protein S3 & 1 & rps3 \\
\hline \multirow[t]{2}{*}{ Predicted gene } & ORF in intronic region & 8 & orf1,orf2,orf3,orf4, orf7, orf8,orf9, orf10 \\
\hline & $\begin{array}{l}\text { ORF in intergenic } \\
\text { region }\end{array}$ & 4 & orf5,orf6,orf11,orf12 \\
\hline Non-coding & Ribosomal RNA & 2 & rns, rnl \\
\hline Gene & Transfer RNA & 27 & $\begin{array}{l}\text { tRNA-Gly_01, tRNA-Gly_02, tRNA-Met_03, } \\
\text { tRNA-Ser_04, tRNA-Pro_05, tRNA-Asn_06, } \\
\text { tRNA-Leu_07, tRNA-Glu_08, tRNA-Ser_09, } \\
\text { tRNA-His_10, tRNA-Asp_11, tRNA-Gln_12, } \\
\text { tRNA-Thr_13, tRNA-Phe_14, tRNA-Ala_15, } \\
\text { tRNA-Met_16, tRNA-Arg_17, tRNA-Cys_18, } \\
\text { tRNA-Trp_19, tRNA-Leu_20, tRNA-Arg_21, } \\
\text { tRNA-Ile_22, tRNA-Ile_23, tRNA-Met_24, } \\
\text { tRNA-Val_25, tRNA-Tyr_26, tRNA-Lys_27 }\end{array}$ \\
\hline Total & & 56 & \\
\hline
\end{tabular}

Additional file 12: Table S6). Three InDels on rps3 were distributed closely $(26,970$ to $26,987 \mathrm{bp})$ and were predicted to be high-risk variants. Two genes, rps3, and nad4L, were selected to verify the variants based on two stains, HM10 and HM54. In rps3, a continuous point mutation (ACCCC/TTGCG), a $9 \mathrm{bp}$ deletion (TTTGGGGAG), and an InDel/SNP (C/ ATAGC,) were detected based on three pairs of PCR primers (Additional file 13: Table S7). In nad4L, a single nucleotide InDel $(\mathrm{C} / \mathrm{CA})$ was detected $(\mathrm{C} / \mathrm{CA})$ (Additional file 6: Figure S6). These results were consistent with the resequencing analysis results, confirming the variants among the mt DNA genomes and thus supporting the conclusion that these genes were under purifying selection.

\section{Comparisons with other fungal $\mathrm{mt}$ genomes}

To study the evolution of $\mathrm{mt}$ in major fungal groups, 15 conserved mt protein-coding genes (atp6, atp8, atp9, cox1, cox2, cox3, nad1, nad2, nad3, nad4, nad4L, nad5, nad6 and $c o b$ ) were collected from 26 representative fungal species from the NCBI database (Additional file 8: Table S2) for phylogenetic analysis. A phylogenetic tree was constructed using the maximum likelihood method (Additional file 1: Figure S1). All nodes in the tree have bootstrap values of $100 \%$, indicating the robustness of the computed tree. The topology of the tree was consistent with the phylogenetic tree constructed using homologous genes with single copies from the whole genome of these species (15 of 27 fungi have been published on NCBI) (Additional file 2: Figure S2), which was supported by the classic taxonomic classification of these fungal species [21].

Molecular clock analysis based on two fossil calibration points (Fig. 3) and the phylogenetic tree shows that Ascomycetes mitochondria started to diverge earlier than that of Basidiomycetes. Agaricomycotina, Pucciniomycotina, and Ustilaginomycotina all had similar divergent evolution in Basidiomycota (Additional file 1: Figure S1); in Agaricomycotina, the genesis of Agaricales was later than Aphyllophorales. H. marmoreus and T. matsutake were estimated to have diverged 72.55 MYA, and genetically were the closest among the 27 species. The comparison of gene order shows that the collinearity level of the 15 conserved $\mathrm{mt}$ genes did not correlate with the phylogenetic distribution of the 27 fungal species and high gene order variability (Fig. 3). For example, collinearity between $P$. pastoris and $C$. albicans were observed to be higher than that of T. cingulata and G. lucidum. Also, the rps3 gene was absent in the mt genome of different phylogenetic groups including $P$. ostreatus, $P$. eryngii, T. cingulate, P. eibomiae, T. indica, T. walkeri, $N$. crassa, C. militaris, and P. pastoris, suggesting that rps3 was less conserved in the mt genome of fungi. Consistent with a previous study [1], two sets of genes nad4L,nad5, and nad2,nad3 were found in tandem in all species. These two sets of genes were further observed to be physically located together with $1 \mathrm{bp}$ overlap between the two genes (Additional file 14: Table S8). 


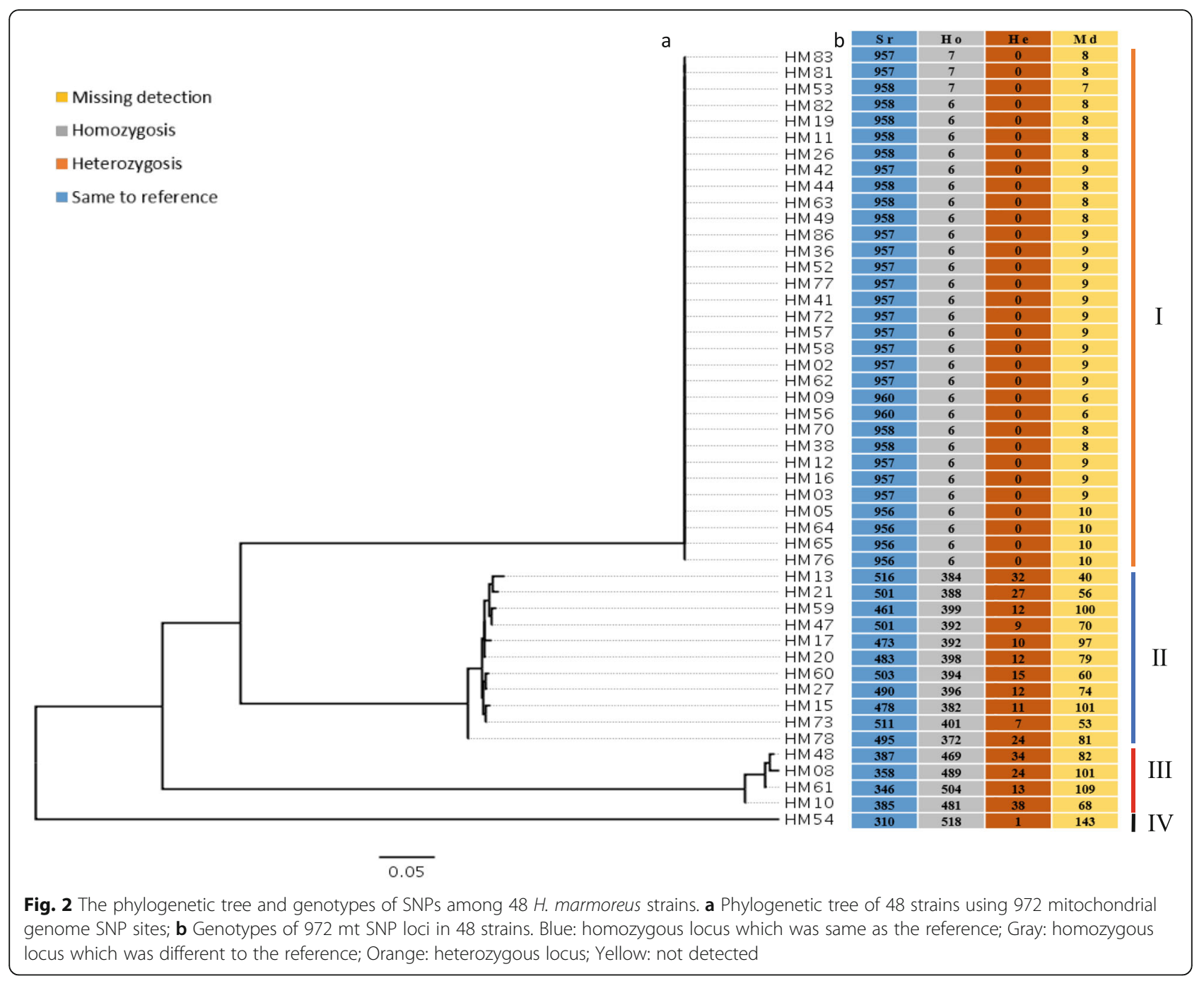

Table 2 Statistics for the effects of variants

\begin{tabular}{|c|c|c|c|c|}
\hline Region of variants & Effect of variants & Impact level & Count & Percent \\
\hline \multirow[t]{7}{*}{ Exon } & Frameshift variant & High & 4 & $0.21 \%$ \\
\hline & Stop lost & High & 1 & $0.05 \%$ \\
\hline & Conservative inframe deletion & Moderate & 1 & $0.05 \%$ \\
\hline & Conservative inframe insertion & Moderate & 1 & $0.05 \%$ \\
\hline & Missense variant & Moderate & 18 & $0.93 \%$ \\
\hline & Synonymous variant & Low & 50 & $2.58 \%$ \\
\hline & Non coding transcript exon variant & Modifier & 116 & $5.98 \%$ \\
\hline Intron & Intron variant & Modifier & 139 & $7.16 \%$ \\
\hline \multirow[t]{3}{*}{ Intergenic } & Intergenic region & Modifier & 1189 & $61.26 \%$ \\
\hline & 500 bp upstream of gene & Modifier & 210 & $10.82 \%$ \\
\hline & 500 bp downstream of gene & Modifier & 212 & $10.92 \%$ \\
\hline Total & - & - & 1941 & $100.00 \%$ \\
\hline
\end{tabular}




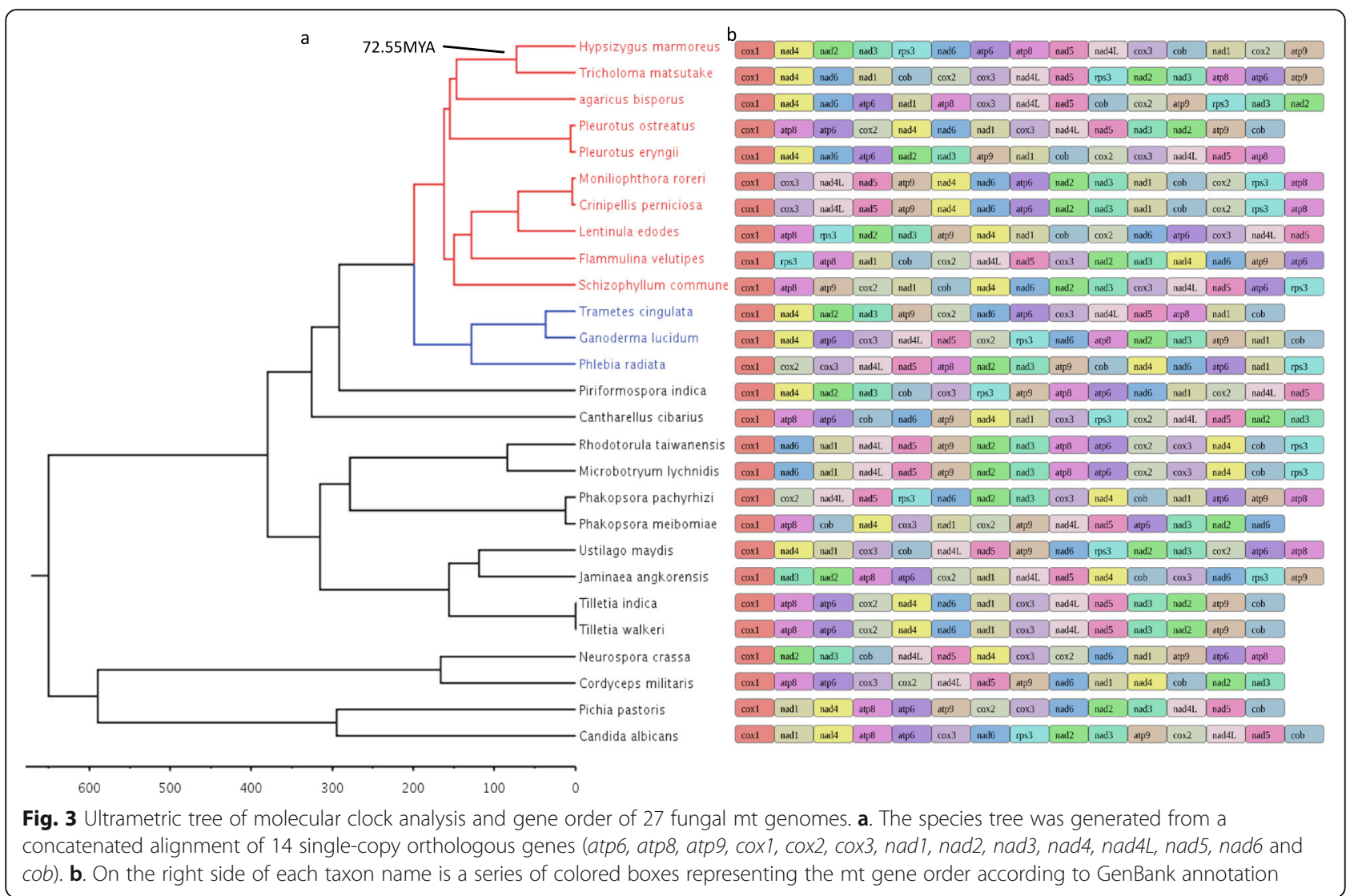

\section{Discussion}

Compared to nuclear DNA, mt DNA is much more susceptible to damage and mutations [22]. Mt genomes are very useful genomic resources in evolutionary biology and systematic studies $[23,24]$ because they evolve faster than nuclear genomes $[11,25]$, and thus mt genome sequences could be evidence for species determination and classification. In this study, we assembled the first complete mt genome of $H$. marmoreus based on the
PacBio sequencing technology. Similar to the mitochondrion of other fungi, the 102,752 bp mtDNA of $\mathrm{H}$. marmoreus contained 15 protein-coding genes, 2 rRNAs, 27 tRNAs and 12 ORFs in intrinsic sequences and intergenic regions. In comparison to the 26 fungi species, the gene content of the $H$. marmoreus $\mathrm{mt}$ genome is similar, but the gene order exhibits only a limited synteny (Fig. 3), suggesting that extensive genome rearrangements in fungal mt genomes took place. Twenty-seven tRNAs existed in

Table 3 Five predicted high impact variants among $H$. marmoreus strains

\begin{tabular}{|c|c|c|c|c|}
\hline Gene & Location & Reference & Variation & Strain and Genotype \\
\hline rps3 & 26970 & CCCCCA & C & $\begin{array}{l}\mathrm{HM} 13[1 / 1]^{\mathrm{A}}, \mathrm{HM} 15[1 / 1], \mathrm{HM} 17[1 / 1], \mathrm{HM} 20[1 / 1], \mathrm{HM} 21[1 / 1], \mathrm{HM} 27[1 / 1] \\
\mathrm{HM} 47[1 / 1], \mathrm{HM} 54[1 / 1], \mathrm{HM} 59[1 / 1], \mathrm{HM} 60[1 / 1], \mathrm{HM} 73[1 / 1], \mathrm{HM} 78[1 / 1]\end{array}$ \\
\hline rps3 & 26981 & TTGGG & T & $\begin{array}{l}\mathrm{HM} 13[1 / 1], \mathrm{HM} 15[1 / 1], \mathrm{HM} 17[1 / 1], \mathrm{HM} 20[1 / 1], \mathrm{HM} 21[1 / 1], \mathrm{HM} 27[1 / 1] \\
\mathrm{HM} 47[1 / 1], \mathrm{HM} 54[1 / 1], \mathrm{HM} 59[1 / 1], \mathrm{HM} 60[1 / 1], \mathrm{HM} 73[1 / 1], \mathrm{HM} 78[1 / 1]\end{array}$ \\
\hline rps3 & 26987 & A & ATAGC,C & $\mathrm{HM} 08[0 / 1]^{\mathrm{B}}, \mathrm{HM} 10[1 / 1], \mathrm{HM} 48[1 / 1], \mathrm{HM} 61[1 / 1]$ \\
\hline nad5 & 67784 & A & T & HM08[1/1],HM10[1/1],HM48[1/1],HM54[1/1],HM61[1/1] \\
\hline nad4L & 71212 & C & CA & $\begin{array}{l}\mathrm{HM} 02[1 / 1], \mathrm{HM} 03[1 / 1], \mathrm{HM} 05[1 / 1], \mathrm{HM} 08[1 / 1], \mathrm{HM} 09[1 / 1], \mathrm{HM} 10[1 / 1], \\
\mathrm{HM} 11[1 / 1], \mathrm{HM} 12[1 / 1], \mathrm{HM} 13[1 / 1], \mathrm{HM} 5[1 / 1], \mathrm{HM} 6[1 / 1], \mathrm{HM} 17[1 / 1], \\
\mathrm{HM} 19[1 / 1], \mathrm{HM} 20[1 / 1], \mathrm{HM} 21[1 / 1], \mathrm{HM} 26[1 / 1], \mathrm{HM} 27[1 / 1], \mathrm{HM} 36[1 / 1], \\
\mathrm{HM} 38[1 / 1], \mathrm{HM} 41[1 / 1], \mathrm{HM} 42[1 / 1], \mathrm{HM} 44[1 / 1], \mathrm{HM} 47[1 / 1], \mathrm{HM} 48[1 / 1], \\
\mathrm{HM} 49[1 / 1], \mathrm{HM} 52[1 / 1], \mathrm{HM} 53[1 / 1], \mathrm{HM} 54[1 / 1], \mathrm{HM} 56[1 / 1], \mathrm{HM} 57[1 / 1], \\
\mathrm{HM} 58[1 / 1], \mathrm{HM} 59[1 / 1], \mathrm{HM} 60[1 / 1], \mathrm{HM} 61[1 / 1], \mathrm{HM} 63[1 / 1], \mathrm{HM} 64[1 / 1], \\
\mathrm{HM} 65[1 / 1], \mathrm{HM} 70[1 / 1], \mathrm{HM} 72[1 / 1], \mathrm{HM} 33[1 / 1], \mathrm{HM} 76[1 / 1], \mathrm{HM} 77[1 / 1], \\
\mathrm{HM} 78[1 / 1], \mathrm{HM} 81[1 / 1], \mathrm{HM} 82[1 / 1], \mathrm{HM} 83[1 / 1]\end{array}$ \\
\hline
\end{tabular}


the Mt genomes of $H$. marmoreus and are thought to contribute to $\mathrm{mt}$ genome rearrangements as the distribution of tRNAs can change location (Perseke et al. 2008) and associate with breakpoints involved in nuclear chromosomal rearrangements in fungi (Di Rienzi et al. 2009). These 56 genes in the mitochondria of $H$. marmoreus are divided into four clusters (Fig. 1), in which the genes were consistently distributed with either the sense or negative sense strand. This phenomenon also existed in the other examined fungal mitochondria (Additional file 5: Figure S5), suggesting that the phenomenon originated before the origin of fungal species. GC content is an important indicator of genome characteristics, which is related to genetic characteristics and has an important influence on the stability of double-stranded DNA. Invertebrate nuclear genomes, the GC content is significantly positively correlated with genome size [26]. However, in this study, the genome sizes varied from $30 \mathrm{~kb}$ to $156 \mathrm{~kb}$, but GC content and genome size showed no significant positive correlation in the $27 \mathrm{ex}$ amined fungal mitochondria, indicating that the genome sizes were independent of GC content in fungal mitochondria.

In this study, we resequenced the mt genomes of 48 $H$. marmoreus strains. The conspecific phylogenetic analysis revealed that the $48 \mathrm{H}$. marmoreus strains might have originated from three ancestors (Fig. 2). The majority of strains were distributed in group I, suggesting that the diversity of the $\mathrm{mt}$ genome in H. marmoreus is low. The H. marmoreus strains from the wild are few and the majority of them are from artificial domestication and hybridization $[27,28]$. The $\mathrm{mt}$ genomes provide the evidence for the origin of the female parent. In this study, the $48 \mathrm{H}$. marmoreus stains were phylogenetically distributed in four clusters with HM54 as the out-group, and these four clusters showed different levels of divergence. Probably, the $H$. marmoreus mt genomes were artificially selected from wild species (HM54), and the divergence rate varied among the four clusters of $H$. marmoreus. Moreover, according to the classification of the cultivated species on the tree, the H. marmoreus was divided into three groups (group I, II, III) and HM54, group I, which can be distinguished from the group II and group III strains by polyacrylamide gel electrophoresis (PAGE) experiments (Additional file 4: Figure S4). This phenomenon is more common in edible fungi cultivars of $H$. marmoreus, so the variety can be identified by the molecular markers.

Previous studies have suggested that repeat sequences in the intergenic regions have the strongest correlation with gene order; the distribution of tRNAs contributes to protein-coding gene order variation among fungi as they can change location, with the "tandem-duplicationrandom-loss" being the model for explaining gene order changes $[5,29,30]$. Here, we have shown that there is high variability in terms of how the 15 protein-coding genes are ordered in the mt genome among Basidiomycetes and Ascomycetes, particularly in basidiomycetes, suggesting a complex interplay of opposing evolutionary forces (Fig. 3). The differentiation of gene order related to divergence time, and the effect of multicollinearity among interspecific species were obvious in the model within a similar differentiation period, but there are also some exceptions, for example, $P$. osteratus/P. eryngii, $P$. taiwanensi/P. meibomiae, and these results were consistent with the previous report [8].

In this study, similar to C. militaris [14], C. albicans [31] and L. kluyveri [32], the H. marmoreus nucleotide variability at intergenic regions ( $\sim 83 \%$ of the whole $\mathrm{mt}$ genome) was higher than intronic and exon genic regions $(\sim 17 \%$ of the whole mt genome) (Table 2), supporting the hypothesis that most of the variants present in protein-coding genes would be removed by selection [32].

The rps3 gene was reported to be a common feature of the mt genome [33]. It encodes a ribosomal protein, a component of the $40 \mathrm{~S}$ subunit and plays a critical role in the initiation of protein translation [34]. Besides, rps3 had extra-ribosomal activities such as DNA repair, cell signaling, apoptosis/survival and transcriptional regulation $[7,35]$. In the 27 fungi species, the rps3 gene was short of the mt genome of $P$. ostreatus, $P$. eryngii, T. cingulate, P. eibomiae, T. indica, T. walkeri, N. crassa, C. militaris and P. pastoris (Fig. 3). In the bioinformatics analysis of variations, three high frameshift variants were found on the rps3 gene and the lowly conserved rps3 was susceptible to high-risk variations (Table 3). The proportion of non-synonymous and synonymous mutations in the rps3 gene was $1.4(\mathrm{dN} / \mathrm{dS}>1)$, which indicates that the gene sequence was positively selected for and that the gene recently evolved, this being of great significance to species evolution [36, 37]. We also verified one insertion (A/ATAGC, C) using PCR product sequencing in HM10 generating the same result. In the HM54 strain, PCR product sequencing also verified that a $9 \mathrm{bp}$ deletion (TTTGGGAG) and multi-point continuous mutation (ACCCC/TTGCG) occurred between positions 26,975 and 26,989 of the $\mathrm{mt}$ genome (Additional file 6: Figure S6). The results suggest a display of unique evolutionary characteristics in $H$. marmoreus as rps 3 is under positive selection. The study argues that it was in part due to elevated rates of evolution in rRNA genes, protein-coding genes were commonly used for the phylogenetic analyses of fungi $[1,11]$. As found in the previous study, when cellular ROS levels increase, the mitochondrial genes are highly vulnerable to DNA damage. Increased ROS induces rps3 accumulation in the mitochondria for DNA repair while significantly decreasing cellular protein synthesis. For the entrance into the mitochondria, the accumulation of rps3 was regulated by interactions with HSP90, HSP70, 
and TOM70 [38]. The specific role of the rps3 gene in DNA damage repair in H. marmoreus and its mechanism requires further study.

Mitochondria, one of the organelles of eukaryotic cells, have their genome and can complete replication, transcription, and translation. In this study, 14 protein-coding genes from the 27 fungi species were used for ML analysis. Combining the ML and Phylogenetic distribution (Fig. 3, Additional file 1: Figure S1, Additional file 8: Table S2) three phylogenetic tree branches according to Basidiomycetes (two branches) and Ascomycetes sub-kingdom are well-supported, which is in agreement with a previous study [39] that found that Agaricomycotina contains 4 orders: Agaricales, Aphyllophorales, Sebacinales and Cantharellales. The previous study was based on a single laccase gene and showed that H.marmoreus and $F$. velutipes were the closest sisters in Agaricales [40]. However, data on single-genes may result in conflicting gene trees and is thus insufficient to reconstruct consistent and accurate phylogenetic hypotheses. Our study based on 14 conserved genes from the $\mathrm{mt}$ genome for the phylogeny of 27 fungi species suggested that $H$. marmoreus was the sister to $T$. matsutake which were estimated to have diverged 72.55 MYA, with the multiple gene scale data solving potential issues with gene trees caused by limited gene sets and therefore providing a more accurate phylogenetic classification [41]. The mt genome does not recombine and has the characteristics of matrilineal inheritance. Cluster analysis of Ciliophora was conducted using mitochondrial and nuclear genome information, and it was found that the clustering of species was not significantly different, but the tree branches were partially different [42]. Here, the single-copy homologous genes of available 15 fungal genomes published on NCBI taken from the 27 fungi were used to construct the phylogenetic trees and the phylogenetic tree constructed by Basidiomycetes and Ascomycetes in previous studies [43], which were found to be consistent with the phylogenetic tree of mitochondria (Additional file 2: Figure S2), indicating that the mt genome could be used for the analysis of the evolutionary development of fungi.

In this study, based on phylogenetic distribution and the time tree estimation, Ascomycetes mitochondria started to diverge earlier than that of Basidiomycetes. A previous study based on six genes estimated that the ancestors of Basidiomycota and Entorrhizomycota split about 530 MYA [44]. Agaricomycotina, Pucciniomycotina, and Ustilaginomycotina all had similar divergent evolutionary time in $\mathrm{Ba}$ sidiomycota which was consistent with previous studies (Additional file 1: Figure S1) [7]. However, our estimation of divergence time was performed on a few calibration points due to the availability of very limited fossil evidence, as the reason, we did not emphasize the divergence time in the Dikarya. Further analysis based on calibration points with more fossil evidence would be essential for the prediction of divergence time in Dikarya in the future.

\section{Conclusions}

In this study, we provided the first $H$. marmoreus reference sequence of the mitochondrial $(\mathrm{mt})$ genome with a circular structure. Comparative analysis of the $\mathrm{mt}$ genomes from 27 fungal species in the dikarya revealed that H. marmoreus was a sister to T. matsutake and Ascomycetes mitochondria started to diverge earlier than that of Basidiomycetes. The correlation between the gene order and divergence time in the fungi $\mathrm{mt}$ genomes were observed among the fungal species. Fourteen of fifteen conserved protein-coding genes of mtDNA were revealed to be under purifying selection in the examined fungal species, suggesting the rapid evolution was caused by positive selection of this gene. Moreover, the phylogeny and variation sited type statistics based $48 \mathrm{H}$. marmoreuss trains provide clear-cut evidence for the existence of four welldefined cultivations isolated lineages, suggesting female ancestor origin of $H$. marmoreus. Our study provides the foundation work for assessing diversity and origin of $H$. marmoreus and its evolution in Dikarya.

\section{Methods \\ Fungal strains and DNA preparation}

A total of $48 \mathrm{H}$. marmoreus dikaryotic strains (Additional file 7: Table S1) were collected from scientific research institutes, universities, and enterprises. The monocytic strain FQX_MS01 was cultured from a spore of strain HM62. All of these strains were cultured in potato dextrose broth at $25^{\circ} \mathrm{C}$ and dark condition for 10-15 days. The mycelia were harvested and washed with sterile deionized water three times, and stored at $-80^{\circ} \mathrm{C}$ before processing for DNA extraction. Finally, freeze-dried mycelia were ground with liquid nitrogen and whole genomic DNA extraction was performed using the CTAB method as previously described by Manicon et al. [45].

\section{Sequencing and assembling of $\mathrm{mt}$ genome}

We sequenced the whole genome of $H$. marmoreus strain FQX_MS01 using the single-molecule sequencing platform Pacbio RSII (Genomics and Biotechnology Research Center, Fujian Agriculture and Forestry University, Fuzhou, China), producing $\sim 100 x$ Pacbio raw data. Canu [46] was then used for de novo genome assembly, obtaining 51 contigs with a total size of 43,691,898 bp and N50 $=1,760,684$ bp. Mt contig was picked out from assembly results through the comparison with mt DNA of Pleurotus ostreatus using BLASTX. Some small InDels mistakes were corrected by mapping short reads from Illumina sequencing on raw genome. The mt contig was assembled into a circle molecule based on the overlap of contig ends.

\section{Mitochondrial genome annotation}

The Mfannot web tool (http://megasun.bch.umontreal. $\mathrm{ca} / \mathrm{RNAweasel} /$ ) was used for the annotation of the $\mathrm{mt}$ 
genome. Meanwhile, cDNA sequences collected from RNA-Seq were aligned to the mt genome by PASA [47], and $\mathrm{mt}$ homologous proteins were aligned to the $\mathrm{mt}$ genome by Genewise. The tRNA genes were identified by tRNAScan-SE [48], and the rRNA genes were identified by the Rfam [49] database. The mt genomic annotation results were manually corrected using the Jbrowse [50] genome browser and WebApollo [51]. OGDRAW [52] and Circos [53] were used to draw the circular map of $\mathrm{mt}$ genome.

\section{Comparative genomics and phylogenetic analysis}

The $26 \mathrm{mt}$ assemblies were downloaded from the NCBI database (https://www.ncbi.nlm.nih.gov/genome/organelle/ ) (Additional file 8: Table S2). Fourteen fully conserved $\mathrm{mt}$ proteins, including 4 cytochrome c-oxidases subunits (cox1, $c o x 2$, cox3 and $c o b$ ), three ATP synthase subunits (atp6, atp8 and atp9) and $7 \mathrm{NADH}$ dehydrogenase subunits (nad1, nad2, nad3, nad4, nad4L, nad5 and nad6) from $H$. marmoreus and the 26 fungal species were used for comparative analysis (Additional file 8: Table S2). Firstly, the homologous protein sequences of all the 27 species were aligned using MAFFT software [54]. Each homologous protein had one alignment result, and all 15 alignments were integrated by joining all sequences into one line for each species. Secondly, the conserved blocks of the alignment were extracted by GBlocks [55]. Thirdly, the best model of protein evolution was determined by ProtTest [56] software. Finally, Maximum likelihood topology searches were completed with RAxML8.1.24 [57] using the model "PROTGAMMALGX", and analysis was conducted with 1000 bootstrap replicates. Two fossil calibration points were fixed in the molecule clock analysis: the most recent common ancestor (MRCA) of Agaricus bisporus and Schizophyllum commune diverged 162 MYA [38]; the MRCA of Candida albicans and Cordyceps militaris diverged 590 MYA [58]. The divergence time of other nodes was calculated by r8s v1.80 [59] software with TN algorithm, PL method and the smoothing parameter value set to 1000 through cross-validation.

\section{Analysis of strain-specific variants}

Each of the 48 dikaryotic strains was sequenced with the Illumina Hiseq2500 platform from an Illumina paired-end library with an insert size of $\sim 450 \mathrm{bp}$, producing $150 \mathrm{bp}$ short reads of up to $\sim 3 \mathrm{~Gb}$ of raw data. The complete raw data for the genome resequencing of the 48 strains were preprocessed by Trimmomatic [60] and aligned to the mtgenome sequence of $H$. marmoreus strain FQX_MS01 by Bowtie2 [61]. GATKv3.6 and Samtoolsv1.3.1 [62] were both used for SNP and InDel detection with default parameters separately, and the $\mathrm{mt}$ variants were selected for this research. Finally, the intersection of the two methods was picked out for a downstream hard filtering with
GATK thresholds: QUAL $>=60, \mathrm{QD}>=10.0, \mathrm{MQ}>=$ 13.0, FS $<=20.0$, MQRankSum $>3.0$ and ReadPosRanSum $>=-3.0$, resulting in 972 reliable SNPs and 401 InDels for the next annotation by SnpEff [63]. The alignment of 48 sequences each with lengths of $972 \mathrm{bp}$ was extracted from the 972 SNP sites, and inputted into RAxML v8.1.24 with the parameter "-m GTRCAT" for the construction of a conspecific phylogenetic tree, and bootstrap analysis was conducted with 1000 times resampling. Primer3 software was used to design primers based on mutation site information (Additional file 13: Table S7), with PAGE and Sanger sequencing being used for validation.

\section{Supplementary information}

Supplementary information accompanies this paper at https://doi.org/10. 1186/s12864-019-6133-z.

Additional file 1: Figure S1. Phylogenetic analysis of $\mathrm{H}$. marmoreus and the other 26 fungal species based on protein sequences of 14 conserved mitochondrial orthologous genes. A max likely hood species tree of 27 fungal species was constructed using RAxML and a bootstrap analysis with 1000 replications was performed. All of the bootstrap values at any node were $100 \%$. The order, sub-kingdom, and kingdom corresponding to each species show their taxonomic classifications.

Additional file 2: Figure S2. Phylogenetic analysis of $\mathrm{H}$. marmoreus and 14 other fungal species based on a single copy of a homologous gene. A maximum likely hood species tree of 15 fungal species was constructed using RAxML and a bootstrap analysis with 1000 replications was performed. All of the bootstrap values at any node were 100\%.

Additional file 3: Figure S3. The phylogenetic and SNP analyze of $H$. marmoreus intraspecific. a. Phylogenetic tree construction of $48 \mathrm{HM}$ strains using 972 mitochondrial genome SNP sites; b. Genotypes of 972 mitochondrial SNP loci in $48 \mathrm{HM}$ strains, calculated as diploid. Yellow: the loci are homozygous and consistent with the reference genome; blue: loci are homozygous and inconsistent with the reference genome; red: loci are heterozygous; green: DNA sequencing reads are not aligned at this locus result.

Additional file 4: Figure S4. PAGE diagram of PCR products for two InDdels markers in different $H$. marmoreus strains. (A) InDel (GGGGTC CCGTAC/G) located at position 93,608 on the mt genomes; (B) InDel (TAGTAA/T) located at position 93,344 on themt genomes. The strains HM44, HM70 and HM86 were the same as HM62 (references genome) in group I, while, strains HM13, HM8, HM48, HM10 and HM54 were classified in group II, and III. $M=$ marker.

Additional file 5: Figure S5. Circle diagrams representing the mitochondrial genomes of 4 different strains.

Additional file 6: Figure S6. PCR product sequencing of the variant sites between HM62 and HM10 strains. (A) At 26987 location on the mt genome, there is a $4 \mathrm{bp}$ InDels in rps3 of HM10. Rps3-2-HM10 and rps3HM10 are two primers of PCR products. (B) At position 26,975 on the $\mathrm{mt}$ genome there is a $4 \mathrm{bp}$ continuous point mutation (ACCCC/TTCGC) and 9 bp deletion (TTGGGGAG) in rps3 of HM54. rps3-HM54 and Rps3-2HM54 are two primers of PCR products. (C) At position 71,212 on the mt genome, there is a $2 \mathrm{bp}$ InDels (C/CA) in nad4L of HM10. Nad4L-10 and Nad4L-2-10 are two primers of PCR products.

Additional file 7: Table S1. Source of the $48 \mathrm{H}$. marmoreus strains. Additional file 8: Table S2. Statistics for the general features of $\mathrm{mt}$ genomes among 26 fungal species.

Additional file 9: Table S3. Variation site statistics for the mt genome of H. marmoreus.

Additional file 10: Table S4. Statistics for the genotypes of 48 strains 
Additional file 11: Table S5. SNPs and genotypes of $48 \mathrm{H}$. marmoreus strains.

Additional file 12: Table S6. Effects of variants on $15 \mathrm{mt}$ conserved genes.

Additional file 13: Table S7. Primers used for $H$. marmoreus DNA amplification and sequencing.

Additional file 14: Table S8. The distances between nad2, nad3 and between nad4L, nad5.

\section{Abbreviations}

ATP: Adenosine triphosphate; bp: Base Pair; dN: Non-synonymous; dS: Synonymous; GATK: Genome Analysis ToolKit; MAFFT: Multiple Alignment using Fast Fourier Transform; MRCA: Most recent common ancestor; MYA: Millions of years ago; NADH: Nicotinamide adenine dinucleotide; ORF: Open Reading Frame; PAGE: Polyacrylamide gel electrophoresis; Rfam: RNA families; rps3: Ribosomal Protein S3; SNPs: Single nucleotide polymorphism

\section{Acknowledgements}

We thank Lianfu Chen for his advice and help in data analysis.

\section{Authors' contributions}

$J Z, G W, R M$, and $J L$ conceived and designed experiments for the manuscript. $G W, J L, Y S, X C, M D, Y D, Y W, L G$, isolated the strain of $H$. marmoreus, performed the genome sequencing. WW, YS and GW performed the bioinformatics. JZ and GW wrote the paper. All authors read and approved the final manuscript

\section{Funding}

This work was supported by the Science and Technology Major Project of Fujian Province [2016NZ0001], the Program for New Century Excellent Talents in Fujian Province [KLa17073A], Agricultural technology extension service system for edible fungus industry in Fujian, China [KNJ-153011-1], and Natural Science Foundation of China [31670021]. These funding bodies had no role in the design of the study, collection, analysis, and interpretation of data, or in writing the manuscript.

\section{Availability of data and materials}

The $\mathrm{mt}$ genome sequences of $\mathrm{H}$. marmoreus have been deposited at GeneBank under the accession number of: HM382825 (https://www.ncbi.nlm nih.gov/nuccore/MH382825.1/).The original genome data was uploaded to NCBI BioProject, under the accession number: PRJNA508399.

\section{Ethics approval and consent to participate}

Not applicable.

\section{Consent for publication}

Not applicable.

\section{Competing interests}

The authors declare that they have no competing interests.

\section{Author details}

${ }^{1}$ Center for Genomics and Biotechnology, Haixia Institute of Science and Technology, Fujian Provincial Key Laboratory of Haixia Applied Plant Systems Biology, College of Life Sciences, Fujian Agriculture and Forestry University, Fuzhou 350002, China. ${ }^{2}$ College of Life Sciences, Fujian Agriculture and Forestry University, Fuzhou 350002, China. ${ }^{3}$ College of Crop Science, Fujian Agriculture and Forestry University, Fuzhou 350002, China. ${ }^{4}$ Department of Plant Biology, University of Illinois at Urbana-Champaign, Urbana, IL 61801, USA

Received: 30 May 2019 Accepted: 23 September 2019

Published online: 22 October 2019

\section{References}

1. Aguileta G, de Vienne DM, Ross ON, Hood ME, Giraud T, Petit E, Gabaldón T. High variability of mitochondrial gene order among fungi. Genome Biol Evol. 2014;6(2):451-65.
2. Palmer JD, Adams KL, Cho Y, Parkinson CL, Qiu Y, Song K. Dynamic evolution of plant mitochondrial genomes: Mobile genes and introns and highly variable mutation rates. Proc Natl Acad Sci U S A. 2000;97(13):6960.

3. Sloan DB, Alverson AJ, Chuckalovcak JP, Wu M, Mccauley DE, Palmer JD, Taylor DR. Rapid Evolution of Enormous, Multichromosomal Genomes in Flowering Plant Mitochondria with Exceptionally High Mutation Rates. PLoS Biol. 2012;10(1):e1001241

4. Kitazaki K, Kubo T. Cost of having the largest mitochondrial genome: evolutionary mechanism of plant mitochondrial genome. J Botany. 2010;2010:5950.

5. Perseke $M$, Fritzsch $G$, Ramsch $K$, Bernt M, Merkle D, Middendorf M, Bernhard D, Stadler PF, Schlegel M. Evolution of mitochondrial gene orders in echinoderms. Mol Phylogenet Evol. 2008;47(2):855-64.

6. Martin M, Matthias B. A method for computing an inventory of metazoan mitochondrial gene order rearrangements. BMC Bioinformatics. 2011;12(Suppl 9):S6.

7. Lin R, Liu C, Shen B, Bai M, Ling J, Chen G, Mao Z, Cheng X, Xie B. Analysis of the complete mitochondrial genome of Pochonia chlamydosporia suggests a close relationship to the invertebrate-pathogenic fungi in Hypocreales. BMC Microbiol. 2015;15(1):5.

8. Zhang S, Wang XN, Zhang XL, Liu XZ, Zhang YJ. Complete mitochondrial genome of the endophytic fungus Pestalotiopsis fici: features and evolution. Appl Microbiol Biotechnol. 2017:101(4):1593-604.

9. Pramateftaki PV, Kouvelis VN, Lanaridis P, Typas MA. The mitochondrial genome of the wine yeast Hanseniaspora uvarum: a unique genome organization among yeast/fungal counterparts. FEMS Yeast Res. 2006;6(1):77-90.

10. Losada L, Pakala SB, Fedorova ND, Joardar V, Shabalina SA, Hostetler J, Pakala SM, Zafar N, Thomas E, Rodriguez-Carres M. Mobile elements and mitochondrial genome expansion in the soil fungus and potato pathogen Rhizoctonia solani AG-3. FEMS Microbiol Lett. 2014:352(2):165-73.

11. Mardanov AV, Beletsky AV, Kadnikov W, Ignatov AN, Ravin NV. The 203 kbp Mitochondrial Genome of the Phytopathogenic Fungus Sclerotinia borealis Reveals Multiple Invasions of Introns and Genomic Duplications. PLoS One. 2014:9(9):e107536.

12. Joardar V, Abrams NF, Hostetler J, Paukstelis PJ, Pakala S, Pakala SB, Zafar N, Abolude OO, Payne G, Andrianopoulos A. Sequencing of mitochondria genomes of nine Aspergillus and Penicillium species identifies mobile introns and accessory genes as main sources of genome size variability. BMC Genomics. 2012;13(1):698.

13. Goddard MR, Burt A. Recurrent invasion and extinction of a selfish gene. Proc Natl Acad Sci U S A. 1999;96(24):13880

14. Zhang S, Hao AJ, Zhao YX, Zhang XY, Zhang YJ. Comparative mitochondrial genomics toward exploring molecular markers in the medicinal fungusCordyceps militaris. Sci Rep. 2017;7:40219.

15. Duò A, Bruggmann R, Zoller S, Bernt M, Grünig CR. Mitochondrial genome evolution in species belonging to the Phialocephala fortinii s.l. - Acephala applanata species complex. Bmc Genomics. 2012;13(1):166.

16. Mui-Keng T, Harsh R, Grant C, Indu S, Zhiliang C, Nandan D, WM R, Vijai G. Characterization of SNP and Structural Variations in the Mitochondrial Genomes of Tilletia indica and Its Closely Related Species Formed Basis for a Simple Diagnostic Assay. Plos One. 2016:11(11):e0166086.

17. Gao X, Wen X, Esser L, Quinn B, Yu L, Yu CA, Xia D. Structural basis for the quinone reduction in the bc1 complex: a comparative analysis of crystal structures of mitochondrial cytochrome bc1 with bound substrate and inhibitors at the qi site. Biochemistry. 2003:42(30):9067-80.

18. Fontanesi F, Soto IC, Horn D, Barrientos A. Assembly of mitochondrial cytochrome c-oxidase, a complicated and highly regulated cellular process. Am J Physiol Cell Physiol. 2006;291(6):1129-47.

19. Li H, Handsaker B, Wysoker A, Fennell T, Ruan J, Homer N, Marth G, Abecasis G, Durbin R. The sequence alignment/map (SAM) format and SAMtools. Bioinformatics. 2009:25(1 Pt 2):1653-4.

20. Ganesan V. Swaiman's pediatric neurology. Eur J Paediatr Neurol. 2012;16(5):566.

21. Hegedusova E, Brejova B, Tomaska L, Sipiczki M, Nosek J. Mitochondrial genome of the basidiomycetous yeast Jaminaea angkorensis. Curr Genet. 2014;60(1):49-59.

22. Mikhail A, Inna S, Glenn W, Susan LD. The maintenance of mitochondrial DNA integrity--critical analysis and update. Cold Spring Harb Perspect Biol. 2013;5(5):a012641

23. Pantou MP, Kouvelis VN, Typas MA. The complete mitochondrial genome of the vascular wilt fungus Verticillium dahliae : a novel gene order for Verticillium and a diagnostic tool for species identification. Curr Genet. 2006:50(2):125-36.

24. Kouvelis VN, Sialakouma A, Typas MA. Mitochondrial gene sequences alone or combined with ITS region sequences provide firm molecular criteria for the classification of Lecanicillium species. Mycol Res. 2008;112(7):829-44. 
25. Basse CW. Mitochondrial inheritance in fungi. Curr Opin Microbiol. 2010;13(6):712-9.

26. Vinogradov AE. Genome size and GC-percent in vertebrates as determined by flow cytometry: the triangular relationship. Cytometry. 2015;31(2):100-9.

27. Xu J, Zhang J, Zhang W, Hu K. The novel role of fungal intracellular laccase: used to screen hybrids between Hypsizigus marmoreus and Clitocybe maxima by protoplasmic fusion. World J Microbiol Biotechnol. 2012;28(8):2625-33.

28. Okamura T, Takeno T, Dohi M, Yasumasa I, Hayashi T, Toyoda M, Noda H, Fukuda S, Horie N, Ohsugi M. Development of mushrooms for thrombosis prevention by protoplast fusion. J Biosci Bioeng. 2000;89(5):474.

29. Lynch M, Koskella B, Schaack S. Mutation pressure and the evolution of organelle genomic architecture. Science. 2006;311(5768):1727.

30. Lavrov DV, Boore JL, Brown WM. Complete mtDNA sequences of two millipedes suggest a new model for mitochondrial gene rearrangements: duplication and nonrandom loss. Mol Biol Evol. 2002;19(2):163.

31. Bartelli TF, Ferreira RC, Colombo AL, Briones MR. Intraspecific comparative genomics of Candida albicans mitochondria reveals non-coding regions under neutral evolution. Infect Genet Evol. 2013;14(2):302-12.

32. Jung PP, Anne F, Cyrielle R, Jing H, Joseph S. Mitochondrial Genome Evolution in a Single Protoploid Yeast Species. G3 (Bethesda, Md). 2012;2(9):1103-11.

33. Sethuraman J, Majer A, Iranpour M, Hausner G. Molecular evolution of the mtDNA encoded rps3 gene among filamentous Ascomycetes Fungi with an emphasis on the Ophiostomatoid Fungi. J Mol Evol. 2009;69(4):372-85.

34. Passmore LA, Schmeing TM, Maag D, Applefield DJ, Acker MG, Algire MA, Lorsch JR, Ramakrishnan V. The eukaryotic translation initiation factors elF1 and elF1A induce an open conformation of the $40 \mathrm{~S}$ ribosome. Mol Cell. 2007;26(1):41.

35. Kim Y, Kim HD, Kim J. Cytoplasmic ribosomal protein S3 (rpS3) plays a pivotal role in mitochondrial DNA damage surveillance. Biochim Biophys Acta. 2013;1833(12):2943-52.

36. Clark AG, Glanowski S, Nielsen R, Thomas PD, Kejariwal A, Todd MA, Tanenbaum DM, Civello D, Lu F, Murphy B, et al. Inferring nonneutral evolution from human-chimp-mouse orthologous gene trios. Science (New York, NY). 2003;302(5652):1960.

37. Wyckoff GJ, Wang W, Wu Cl. Rapid evolution of male reproductive genes in the descent of man. Nature. 2000;403(6767):304.

38. Tedersoo L, Bahram M, Ryberg M, Otsing E, Köljalg U, Abarenkov K. Global biogeography of the ectomycorrhizal /sebacina lineage (Fungi, Sebacinales) as revealed from comparative phylogenetic analyses. Mol Ecol. 2014;23(16):4168-83.

39. Jiang L, Yang D, Cao Y, Wang P, Zhang Y, Zhang KQ, Xu J, Zhang Y. The complete mitochondrial genome of the edible Basidiomycete mushroom Phlebopus Portentosus. Mitochondrial DNA Part B. 2017;2(2):696-7.

40. Zhang J, Chen H, Chen M, Ren A, Huang J, Wang H, Zhao M, Feng Z. Cloning and functional analysis of a laccase gene during fruiting body formation in Hypsizygus marmoreus. Microbiol Res. 2015;179(1):54-63.

41. Bullerwell CE, Lang BF. Fungal evolution: the case of the vanishing mitochondrion. Curr Opin Microbiol. 2005;8(4):362-9.

42. Wang $P$, Wang $Y$, Wang C, Zhang T, Al-Farraj SA, Gao F. Further consideration on the phylogeny of the Ciliophora: Analyses using both mitochondrial and nuclear data with focus on the extremely confused class Phyllopharyngea. Mol Phylogenet Evol. 2017;112(undefined):96-106.

43. Chen L, Gong Y, Cai Y, Liu W, Zhou Y, Xiao Y, Xu Z, Liu Y, Lei X, Wang G. Genome Sequence of the Edible Cultivated Mushroom Lentinula edodes (Shiitake) Reveals Insights into Lignocellulose Degradation. PLoS One. 2016;11(8):e0160336.

44. Zhao RL, Li GJ, Sánchez-Ramírez S, Stata M, Yang ZL, Wu G, Dai YC, He SH, Cui BK, Zhou JL. A six-gene phylogenetic overview of Basidiomycota and allied phyla with estimated divergence times of higher taxa and a phyloproteomics perspective. Fungal Divers. 2017;84(1):1-32.

45. Manicom BQ, Barjoseph M, Rosner A, Vigodskyhaas H, Kotze JM. Potential applications of random DNA probes and restriction fragment length polymorphisms in the taxonomy of the fusaria. Phytopathology. 1987;77(5):531-3.

46. Koren S, Walenz BP, Berlin K, Miller JR, Bergman NH, Phillippy AM. Canu: scalable and accurate long-read assembly via adaptive $k$-mer weighting and repeat separation. Genome Res. 2017;27(5):722.

47. Haas BJ, Salzberg SL, Wei Z, Pertea M, Allen JE, Orvis J, White O, Buell CR, Wortman JR. Automated eukaryotic gene structure annotation using EVidenceModeler and the program to assemble spliced alignments. Genome Biol. 2008;9(1):R7.

48. Lowe TM, Chan PP. tRNAscan-SE On-line: integrating search and context for analysis of transfer RNA genes. Nucleic Acids Res. 2016:44(Web Server issue):W54-7.
49. Burge SW, Daub J, Eberhardt R, Tate J, Barquist L, Nawrocki EP, Eddy SR, Gardner PP, Bateman A. Rfam 11.0: 10 years of RNA families. Nucleic Acids Res. 2013;41(Database issue):226-32.

50. Skinner ME, Uzilov AV, Stein LD, Mungall CJ, Holmes IH. JBrowse: a nextgeneration genome browser. Genome Res. 2009;19(9):1630.

51. Buels R, Lee E. WebApollo: a web-based sequence annotation editor for distributed community annotation. In: International Plant and Animal Genome Conference Xxi; 2013.

52. Lohse M, Drechsel O, Kahlau S, Bock R. OrganellarGenomeDRAW—a suite of tools for generating physical maps of plastid and mitochondrial genomes and visualizing expression data sets. Nucleic Acids Res. 2013;41(Web Server issue):W575.

53. Krzywinski M, Schein J, Birol I, Connors J, Gascoyne R, Horsman D, Jones SJ, Marra MA. Circos: an information aesthetic for comparative genomics. Genome Res. 2009;19(9):1639-45.

54. Katoh K, Standley D. MAFFT multiple sequence alignment software version improvements in performance and usability. Mol Biol Evol. 2013;30(4):772-80.

55. Dereeper A, Audic S, Claverie JM, Blanc G. BLAST-EXPLORER helps you building datasets for phylogenetic analysis. BMC Evol Biol. 2010;10(1):8-8.

56. Darriba D, Taboada GL, Doallo R, Posada D. ProtTest 3: fast selection of bestfit models of protein evolution. Bioinformatics. 2011;27(8):1164-5.

57. Stamatakis A. RAxML version 8: a tool for phylogenetic analysis and postanalysis of large phylogenies. Bioinformatics. 2014;30(9):1312-3.

58. Gaya E, Fernández-Brime S, Vargas R, Lachlan RF, Gueidan C, Ramírez-Mejía $\mathrm{M}$, Lutzoni F. The adaptive radiation of lichen-forming Teloschistaceae is associated with sunscreening pigments and a bark-to-rock substrate shift. Proc Natl Acad Sci U S A. 2015:112(37):11600-5.

59. Sanderson MJ. Estimating absolute rates of molecular evolution and divergence times: a penalized likelihood approach. Mol Biol Evol. 2002;19(1):101-9.

60. Bolger AM, Lohse M, Usadel B. Trimmomatic: a flexible trimmer for Illumina sequence data. Bioinformatics (Oxford, England). 2014;30(15):2114.

61. Langmead B, Salzberg SL. Fast gapped-read alignment with bowtie 2. Nat Methods. 2012;9(4):357.

62. Heng L. A statistical framework for SNP calling, mutation discovery, association mapping and population genetical parameter estimation from sequencing data. Bioinformatics. 2011;27(21):2987-93.

63. Cingolani P, Platts A, Wang LL, Coon M, Nguyen T, Wang L, Land SJ, Lu X, Ruden DM. A program for annotating and predicting the effects of single nucleotide polymorphisms, SnpEff: SNPs in the genome of Drosophila melanogaster strain w1118; iso-2; iso-3. Fly. 2012;6(2):80-92.

\section{Publisher's Note}

Springer Nature remains neutral with regard to jurisdictional claims in published maps and institutional affiliations.

Ready to submit your research? Choose BMC and benefit from:

- fast, convenient online submission

- thorough peer review by experienced researchers in your field

- rapid publication on acceptance

- support for research data, including large and complex data types

- gold Open Access which fosters wider collaboration and increased citations

- maximum visibility for your research: over $100 \mathrm{M}$ website views per year

At $\mathrm{BMC}$, research is always in progress.

Learn more biomedcentral.com/submissions 